\title{
Urinary Excretion of Lactate, 2-Oxoglutarate, Citrate, and Glycerol in Patients with Glycogenosis Type I
}

\author{
JOHN FERNANDES AND RUUD BERGER \\ Department of Pediatrics and Laboratory of Developmental Biochemistry of the Department of Pediatrics, \\ University of Groningen, Groningen, The Netherlands
}

\begin{abstract}
Growth retardation and lactic aciduria are well-known abnormalities in patients with a deficiency of either glucose-6-phosphatase or glucose-6-phosphate translocase. In 19 patients with glucose-6-phosphatase and two patients with glucose-6-phosphate translocase, growth retardation was quantified by calculating the height standard deviation score. The urinary excretion of lactate and some other metabolites was quantified by calculating the lactate/creatinine, 2-oxoglutarate/creatinine, citrate/creatinine, and glycerol/creatinine ratios in urine. Significant correlations were found between the lactate/creatinine ratio, the 2-oxoglutarate/creatinine ratio, and height $\mathrm{SD}$ score. Urinary lactate appeared to respond promptly to changes of the diet, while urinary 2-oxoglutarate responded only slowly, as did growth itself. The citrate/creatinine ratio and the glycerol/creatinine ratio were within the normal range and varied little. It was concluded that the urinary 2-oxoglutarate excretion primarily reflects the severity of the disease as expressed in stunted growth. Thus, while urinary lactate levels are more suitable for monitoring the diet, urinary 2-oxoglutarate levels can be used as an indication for intensive treatment with hyperalimentation. (Pediatr Res 21: 279-282, 1987)
\end{abstract}

\section{Abbreviations}

GSD-IA, glycogen storage disease caused by deficiency of glucose-6-phosphatase

GSD-IB, glycogen storage disease caused by deficiency of glucose-6-phosphate translocase

HSDS, height standard deviation score

GDF, gastric drip feeding

Since 1975 we treat our patients with GSD-IA by means of GDF during the night (1). Most patients showed spectacular catch-up growth. Some patients, however, were less sensitive to this treatment, and a few were even resistant. Struck by the fact that two patients of the latter category showed excessive urinary excretion of 2-oxoglutarate besides excretion of lactate, we investigated whether the differences in growth response of the patients in GDF were reflected by differences in the excretion of particular metabolites in the urine.

Lactic aciduria has been found in many inborn errors of carbohydrate metabolism. In GSD-IA the urinary lactate excretion appeared to be a useful parameter to estimate the glucose

Received May 23, 1986; accepted October 23, 1986.

Correspondence John Fernandes, Department of Pediatrics, University Hospital, 59 Oostersingel, 9713 EZ Groningen, The Netherlands. requirement of the patient (2). 2-Oxoglutaric aciduria has been found incidentally in patients with enzyme defects of both the 2oxoglutarate dehydrogenase complex and the pyruvate dehydrogenase complex (3-5) and in one patient with GSD-IA and one patient with GSD-IB (6). Urinary citrate excretion has been studied in various inborn errors of metabolism with inconclusive results $(6,7)$. Citric acid is a major carrier of $\mathrm{C}_{2}$ units from the mitochondria where they are produced from pyruvate during carbohydrate oxidation, to the cytosol, where they are utilized in fatty acid synthesis. Therefore the excretion of citrate in the urine of GSD-I patients may reflect their excessive lipid synthesis. Glycerol was the fourth metabolite whose excretion in urine we determined. Apart from its function in phospholipid and triglyceride synthesis, it is an important substrate for gluconeogenesis (8). It has been found in high concentrations in the urine of a patient with fructose-1,6-diphosphatase deficiency, which blocks gluconeogenesis (9), so hyperexcretion of glycerol might also occur in patients with GSD-IA and IB in case of deficient availability of glucose.

\section{PATIENTS}

We studied 19 patients with GSD-IA and two patients with GSD-IB. The diagnosis was confirmed by enzyme assay of a liver biopsy in all patients except one. In this case, the diagnosis of GSD-IA was inferred from the enzyme defect of his brother. The age of the patients varied from 2 to $16 \mathrm{yr}$. The dietary treatment (10) consisted of frequent meals, sucrose-restricted and polyunsaturated fats-enriched. The composition was approximately $60-$ $65,10-15$, and 25 energy percent for carbohydrates, proteins, and fats, respectively. GDF administered to 19 patients during 10 to $12 \mathrm{~h}$ per night, consisted of a complete infant formula, lactose-restricted, without sucrose and with extra maltodextrin (1). The energy content of GDF contributed approximately 30 to $35 \%$ of the total daily energy intake. In two patients with the most severe growth retardation, an experimental diet was introduced in order to improve growth and metabolic abnormalities. The new diet consisted of a high-energy liquid formula of fixed composition and quantity, administered every hour during $14 \mathrm{~h}$ at daytime as well as by GDF during $10 \mathrm{~h}$ at night. The frequentmeal regime was introduced in order to imitate GDF and benefit from its supposedly favorable effects. This pseudo-GDF diet was preceded by continuous GDF, day and night, during 3 to 4 wk in order to "titrate" the patient's requirements and tolerance to hyperalimentation.

The patients were free from diseases or abnormalities other than due to their primary disease. Kidney function was evaluated at regular intervals. No signs of complete or incomplete Fanconi's syndrome were observed. The height of the patients was expressed as their HSDS (11) calculated from Dutch growth diagrams (12). 


\section{METHODS}

Urine was collected in $12 \mathrm{~h}$ day and night samples by cumulatively freezing each voided urine during the stay of the child in the hospital, or as freshly voided samples both at the end of the night and the day when the child stayed at home. Each urine sample was immediately frozen in the freezer in the hospital, or at the home of the patient and brought to the hospital in the frozen state. Chemical analyses were performed immediately after thawing. Lactate, 2-oxoglutarate, citrate, and glycerol were determined by enzymatic methods (13). Creatinine was determined by an automated assay. Lactate, citrate, and glycerol were found to be essentially stable during storage at $-20^{\circ} \mathrm{C}$. The concentration of 2-oxoglutarate was found to be stable for several weeks, if stored at $-20^{\circ} \mathrm{C}$. However, it decreased by $20-40 \%$, depending on the initial concentration, upon storage at $-20^{\circ} \mathrm{C}$ for more than 6 months. Therefore, this metabolite was determined as soon as possible.

The results of lactate assays of urines obtained at the home of the patient were found to be in agreement with those of the samples obtained in the hospital under steady-state conditions (2). The lactate/creatinine, 2-oxoglutarate/creatinine, citrate/creatinine, and glycerol/creatinine ratios were calculated to correct for variations in diuresis and to facilitate the comparison of patients with different ages. The data obtained from urines sampled twice a day during 8 to 10 successive days were averaged to level out fluctuations in the patient's metabolic state because of unknown dietary irregularities or minor infections.

Correlations between variables were determined by Spearman's correlation test. Wilcoxon's sum of rank test was used to determine significance of differences. Correlation of variables and differences between means were considered to be significant if $p<0.05$ (14).

\section{RESULTS}

The mean lactate/creatinine ratios versus HSDS are presented in Figure 1. Lactic aciduria was observed in almost all 21 patients, even in those with normal growth (HSDS > -1). The higher ratios were found in patients with the lower HSDS values. The predominance of growth-retarded children, with HSDS less than zero, was due to the fact that the sampling period usually was before GDF could have manifested its long-term favorable effect

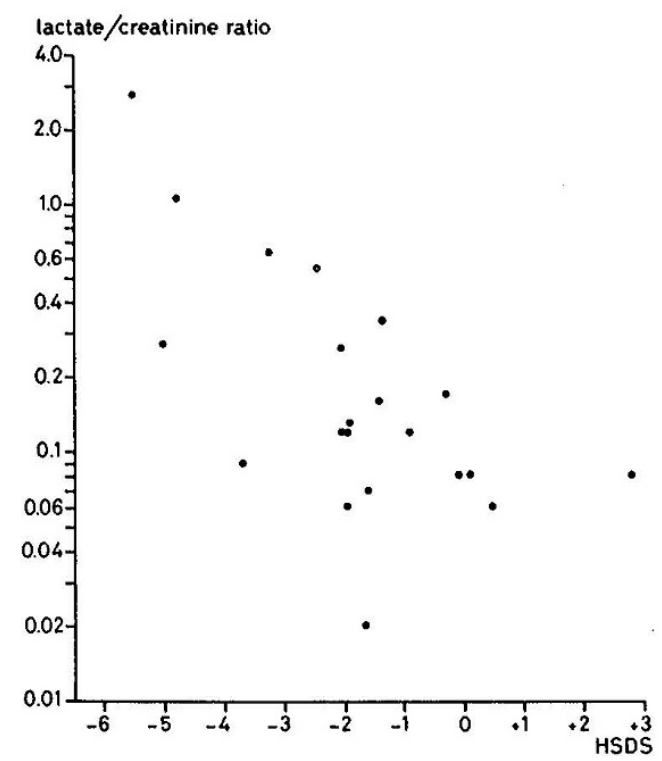

Fig. 1. Mean urinary lactate/creatinine ratio (logarithmic scale) calculated from the assays of urines collected at daytime during the earliest 10 days' sampling period of 19 patients with GSD-IA and two patients with GSD-IB versus their HSDS (linear scale).

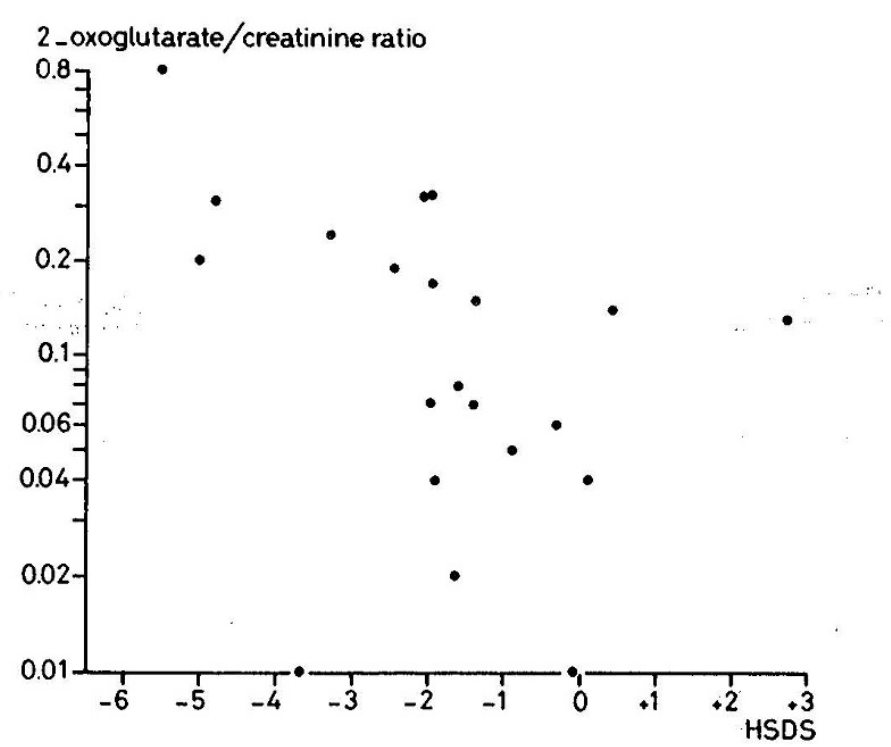

Fig. 2. Mean urinary 2-oxoglutarate/creatinine ratio (logarithmic scale) versus HSDS (linear scale) of the same material as presented in Figure 1.

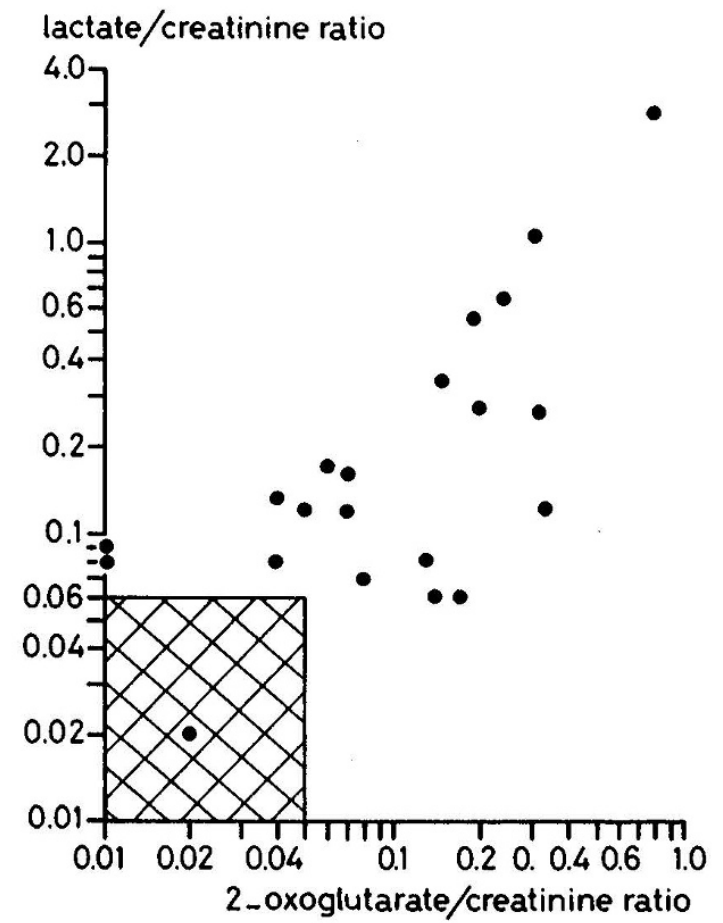

Fig. 3. Mean urinary lactate/creatinine ratio versus mean urinary 2oxoglutarate/creatinine ratio (logarithmic scale for both) of the same material as presented in Figures 1 and 2. The shaded area represents the range of normal children.

on growth (1). The mean 2-oxoglutarate/creatinine ratios versus HSDS are shown in Figure 2. Again, the higher ratios were found in patients with the lower HSDS values. Both lactate/creatinine and 2-oxoglutarate/creatinine ratios were significantly (inversely) correlated with HSDS $(p<0.005$ and $0.025<p<0.05$, respectively).

The mean lactate/creatinine ratios versus mean 2-oxoglutarate/creatinine ratios of all 21 patients are shown in Figure 3. Taking 0.06 as the upper limit of normal lactate/creatinine ratios (15) and 0.05 as the upper limit of normal 2-oxoglutarate/ 
creatinine ratios ( $n=15$, data not shown), an area for the normal ranges was defined. All GSD patients except one were outside this area. The excretions of lactate and 2-oxoglutarate were significantly (positively) correlated $(0.025<p<0.05)$. In normal children there was no correlation ( $p>0.1$, results not shown). To determine whether severe growth retardation was reflected in a great variability of lactate and/or 2-oxoglutarate excretion, the $\mathrm{SD}$ of the geometric mean for each patient was correlated with HSDS. However, no significant correlation, either positively or inversely, was found ( $p>0.1$, results not shown). The mean citrate/creatinine ratios of all 21 GSD patients varied from 0.04 to 0.41 as compared to a range of 0.12 to 0.73 in normal children $(n=15)$, and the mean glycerol/creatinine ratio of all 21 GSD patients varied from 0.01 to 0.12 as compared to a range of 0.005 to 0.03 in normal children $(n=15)$. Both were, therefore, not significantly different from normal. Lactic aciduria is known to be influenced by the amount of glucose administered in GDF: a glucose supply below the theoretically calculated requirement (16) elicits excessive lactate excretion (15). This phenomenon is not known for 2-oxoglutarate excretion. Therefore, the effect of dietary changes on lactate and 2-oxoglutarate excretion was investigated in longitudinal studies in two patients with the most severe growth retardation. An experimental pseudo-GDF was

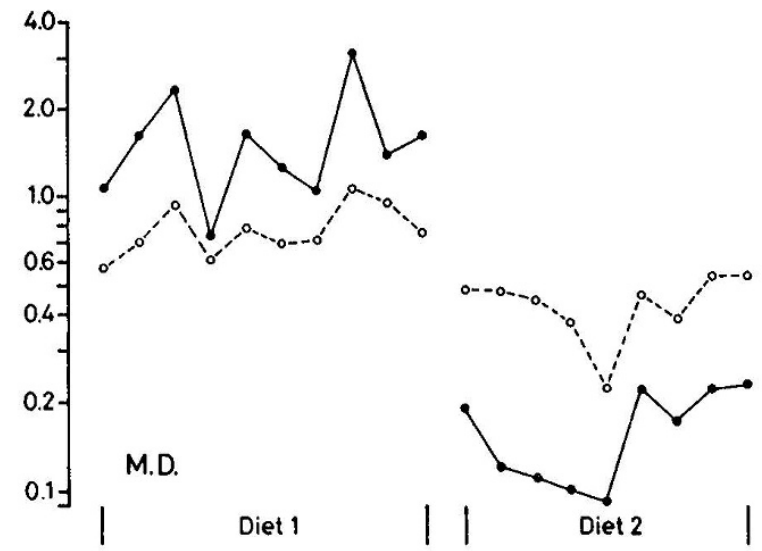

Fig. 4. Lactate/creatinine ratio $(\bullet)$ and 2-oxoglutarate/creatinine ratio $(O)$ of patient MD with GSD-IA. Diet 1 at home; GDF during $12 \mathrm{~h}$, irregular meals at daytime, total energy $2100 \mathrm{kcal} /$ day. Diet 2 in the hospital; GDF during $10 \mathrm{~h}$, pseudo-GDF at daytime, total energy 2330 $\mathrm{kcal} /$ day. introduced in order to improve growth and metabolic abnormalities (see "Patients"). As can be seen from Figure 4, the transition from the routine diet to the pseudo-GDF diet in the first patient was followed by a prompt fall of the lactate/creatinine ratios below the 2-oxoglutarate/creatinine ratios which decreased little. In the second patient, treated in a similar manner (Table 1), the lactate/creatinine ratio decreased considerably during continuous GDF and pseudo-GDF, both compared with the initial routine diet. It reached the same level as the 2oxoglutarate/creatinine ratio, which decreased much less. Again, dietary changes appeared to have a strong effect on the lactate/ creatinine ratio and much less on the 2-oxoglutarate/creatinine ratio. In the latter patient, the mean citrate/creatinine ratio increased during pseudo-GDF, but remained well within the normal range (Table 1).

\section{DISCUSSION}

The excretion in the urine of the metabolites, selected in this study, is determined by two main factors: the rate of production and the renal clearance. Lactate is the end product of glycolysis when the rate of glucose breakdown exceeds the rate of pyruvate oxidation in tissues. In the kidney tubulus lactate is reabsorbed by a specific transporter, shared with ketone bodies and uric acid (17).

2-Oxoglutarate plays a key role in the transport of reduction equivalents from glycolytically generated $\mathrm{NADH}$ across the mitochondrial inner membrane to the respiratory chain via the malate-aspartate cycle (18). The origin of 2-oxoglutaric aciduria in our 21 patients with GSD-IA and GSD-IB has not been clarified in this study. Abnormal kidney tubulus function might result in hyperexcretion of this metabolite, but this should be accompanied by hyperexcretion of citrate, both metabolites sharing a common transport system in the tubulus $(7,17)$. This is not the case, however. Thus, a hepatic origin of 2-oxoglutaric aciduria is much more probable. 2-Oxoglutaric aciduria and lactic aciduria, if present at the same time, might be due to insufficient availability of cofactors, common for both the 2 oxoglutarate dehydrogenase complex and the pyruvate dehydrogenase complex. However, this is difficult to reconcile with the observation that 2-oxoglutaric aciduria may persist, while lactic aciduria almost disappears during hyperalimentation (Fig. 4), as has been found earlier (19). This finding does not tally with the supposition that excessive 2-oxoglutarate excretion would be a second "outlet" of abnormally increased glycogenolysis, excessive lactate excretion being the first. It would be more compatible

Table 1. Effect of continuous GDF or pseudo-GDF on organic aciduria*

\begin{tabular}{|c|c|c|c|c|c|c|}
\hline & \multicolumn{2}{|c|}{$\frac{\text { Lactate } \mathrm{mM}}{\text { creatinine } \mathrm{mM}}$} & \multicolumn{2}{|c|}{$\frac{\text { 2-Oxoglutarate } \mathrm{mM}}{\text { creatinine } \mathrm{mM}}$} & \multicolumn{2}{|c|}{$\frac{\text { Citrate } \mathrm{mM}}{\text { creatinine } \mathrm{mM}}$} \\
\hline & Day & Night & Day & Night & Day & Night \\
\hline Diet 1 & $\begin{array}{c}0.50(0.07-8.98) \\
(28)\end{array}$ & $\begin{array}{c}0.34(0.18-3.76) \\
(29)\end{array}$ & $\begin{array}{c}0.29(0.06-0.61) \\
(31)\end{array}$ & $\begin{array}{c}0.27(0.09-0.50) \\
(31)\end{array}$ & $\begin{array}{c}0.07(0.04-0.14) \\
(28)\end{array}$ & $\begin{array}{c}0.06(0.005-0.11) \\
(27)\end{array}$ \\
\hline Diet 2a & $\begin{array}{c}0.09(0.01-1.25) \dagger \\
(37)\end{array}$ & $\begin{array}{c}0.07(0.002-0.71) \dagger \\
(36)\end{array}$ & $\begin{array}{c}0.11(0.02-0.28) \dagger \\
(41)\end{array}$ & $\begin{array}{c}0.09(0.01-0.40) \dagger \\
(41)\end{array}$ & $\begin{array}{c}0.35(0.02-1.05) \dagger \\
(40)\end{array}$ & $\begin{array}{c}0.23(0.03-0.36) \dagger \\
(38)\end{array}$ \\
\hline Diet $2 b$ & $\begin{array}{c}0.21(0.12-0.28) \dagger \\
(6)\end{array}$ & $\begin{array}{c}0.16(0.08-0.27) \dagger \\
(7)\end{array}$ & $\begin{array}{c}0.11(0.06-0.20) \dagger \\
(7)\end{array}$ & $\begin{array}{c}0.17(0.08-0.19) \dagger \\
(8)\end{array}$ & $\begin{array}{c}0.26(0.15-0.37) \\
(7)\end{array}$ & $\begin{array}{c}0.12(0.04-0.18) \dagger \\
(8)\end{array}$ \\
\hline Normal values & $\begin{array}{c}0.01(0.01-0.03) \\
(5)\end{array}$ & $\begin{array}{c}0.01(0.01-0.03) \\
(5)\end{array}$ & $\begin{array}{c}0.02(0.02-0.04) \\
(5)\end{array}$ & $\begin{array}{c}0.02(0.01-0.02) \\
(5)\end{array}$ & $\begin{array}{c}0.20(0.18-0.28) \\
(5)\end{array}$ & $\begin{array}{c}0.10(0.08-0.15) \\
(5)\end{array}$ \\
\hline
\end{tabular}

* Diets 1, 2a, and $2 \mathrm{~b}$ were administered sequentially to patient AR with GSD-IA. Diet 1 : routine diet with irregular and unequal meals at daytime and GDF at night; total energy $1800 \mathrm{kcal} /$ day. Diet 2a: continuous GDF around the clock; total energy $2200 \mathrm{kcal} / \mathrm{day}$. Diet 2b: pseudo-GDF diet (see text under "Patients"); total energy $2000 \mathrm{kcal} /$ day. Results are given as median and range. Normal values were obtained from age-matched individuals. Numbers of observation are in parentheses.

$\dagger$ Significance of differences (diet $2 \mathrm{a}$ versus diet 1 and diet $2 \mathrm{~b}$ versus diet 1 ): $p<0.01$. 
with an excessive input of other substrates into the tricarboxylic acid cycle, e.g. for gluconeogenesis. A persistently increased excretion of 2-oxoglutarate would then indicate that the patient's requirement for glucose (and other fuels) is not met and that the energy intake should be increased. This is especially the case if the increased 2-oxoglutarate excretion is accompanied by marked growth retardation.

Citrate excretion was not elevated. Rather it appeared to be depressed if the 2-oxoglutarate and lactate excretions were high. The small changes of citrate excretion, reciprocal to those of lactate, might be due to small changes of acid-base equilibrium (20). Glycerol excretion was not elevated and its determination contributed neither to the evaluation of the metabolic status of the patient nor to better treatment.

We conclude that the determination of both lactate and 2oxoglutarate in the urine improves the evaluation of the condition of a GSD patient. The level of lactate excretion reflects mainly the adequacy of the diet, while the level of 2-oxoglutarate excretion reflects both the adequacy of the diet and the severity of the disease as manifested by retarded growth, but particularly the latter. It might be used as an indication for intensive treatment of the patient, for instance with hyperalimentation.

Acknowledgments: The authors thank Mrs. W. Brouwer, Mrs. C. Oldenhof, Mrs. S. A. Stoker-De Vries, Mrs. I. Mulder, and Mr. A. Siemensma for their expert technical assistance. Mrs. G. van Rijn for her help in composing the diets, Dr. V. Fidler for his advice on statistics, Mr. G. Messchendorp for drawing the figures, and Mrs. H. Marra for typing the manuscript.

\section{REFERENCES}

1. Fernandes J, Jansen $H$, Jansen TC 1979 Nocturnal gastric drip feeding in glucose-6-phosphatase deficient children. Pediatr Res 13:225-229

2. Fernandes J, Smit GPA, Berger R 1984 The lactate concentration of the urine, a parameter for the adequacy of dietary treatment of patients with glucose6-phosphatase deficiency. J Inherited Metab Dis 7 (suppl 2):149-150

3. Haworth JC, Perry TL, Blass JP, Hansen S, Urquhart N 1976 Lactic acidosis in three sibs due to defects in both pyruvate dehydrogenase and $\alpha$-ketoglutarate dehydrogenase complexes. Pediatrics 58:564-572

4. Chalmers RA, Lawson MA, Borud O 1977 Gaschromatographic and mass spectrometric studies on urinary organic acids in a patient with congenital lactic acidosis due to pyruvate decarboxylase deficiency. Clin Chim Acta 77:117-124

5. Kuhara $T$, Shinka $T$, Inone $Y$, Matsumoto $M$, Yoshino $M$, Sakaguchi $Y$, Matsumoto I 1983 Studies of urinary organic acid profiles of a patient with dihydrolipoyl dehydrogenase deficiency. Clin Chim Acta 133:133-140

6. Chalmers RA, Ryman BE, Watts RWE 1978 Studies in a patient with in vivo evidence of type I glycogenosis and normal enzyme activities in vitro. Acta Paediatr Scand 67:201-207

7. Kohlschütter A, Behbehani A, Langenbeck U, Albani M, Heidemann P, Hoffmann G, Kleineke J, Lehnert W, Wendel U 1982 A familial progressive neurodegenerative disease with 2-oxoglutaric aciduria. Eur J Pediatr 138:3237

8. Lin ECC 1977 Glycerol utilization and its regulation in mammals. Ann Rev Biochem 46:765-795

9. Dremsek PA, Sacher M, Stögmann W, Gitzelmann R, Bachmann C 1985 Fructose-1,6-diphosphatase deficiency: glycerol excretion during fasting test. Eur J Pediatr 144:203-204

10. Fernandes J, Pikaar NA 1969 Hyperlipemia in children with liver glycogen disease. Am J Clin Nutr 22:617-627

11. Tanner JM, Whitehouse RH, Hughes PCR, Vince FP 1971 Effects of human growth hormone treatment for 1 to 7 years on growth of 100 children, with growth hormone deficiency, low birthweight, inherited smallness, Turner's syndrome and other complaints. Arch Dis Child 46:745-782

12. Wieringen JC van, Roede MJ, Wit JM 1985 Groeidiagrammen voor patiëntenzorg. Tijdschr Kindergeneeskd 53:147-152

13. Bergmeyer HU 1970 Methoden der Enzymatische Analyse, Vol 2. Verlag Chemie, Weinheim

14. Langley R 1968 Practical Statistics. Pan Books Ltd, London

15. Fernandes J, Blom W 1975 Urinary lactate excretion in normal children and in children with enzyme defects of carbohydrate metabolism. Clin Chim Acta 66:345-352

16. Bier DM, Leake RD, Haymond MW, Arnold KJ, Gruenke LD, Sperling MA, Kipnis DM 1977 Measurement of "true" glucose production rates in infancy and childhood with 6,6-dideuteroglucose. Diabetes 26:1016-1023

17. Pitts RF 1968 Physiology of the Kidney and Body Fluids. Year Book Medical Publ Inc., Chicago, pp 81-86

18. Borst P 1963 In: Karlson P (ed) Funktionelle und Morphologische Organisation der Zelle. Springer Verlag, Berlin, pp 137-162

19. Chalmers RA 1984 Organic acids in urine of patients with congenital lactic acidosis: an aid to differential diagnosis. J Inherited Metab Dis 7 (suppl 1):79-89

20. Miller LA, Stapleton FB 1985 Urinary citrate excretion in children with hypercalciuria. J Pediatr 107:263-266 